\title{
Per una intertestualità manzoniana nella Cognizione del dolore di Carlo Emilio Gadda
}

\author{
Fabrizio Rusconi (UFRJ)
}

fabriziorusconi@gmail.com

\section{Resumo:}

O objetivo deste estudo é investigar a questão das fontes "manzonianas" em Carlo Emilio Gadda (1893-1973). Vou tratar especificamente da Cognizione del dolore (obra publicada pela editora Rocco em 1988: título O Conhecimento da Dor), obra-prima de Gadda, atravessada por um poderoso plurilinguismo, bem como por uma inesgotável intertextualidade. Sabemos também que Gadda era um cultor de Manzoni (1785-1873), ao qual, já em Janeiro de 1927, nas páginas da "Solaria" dedicou a própria Apologia manzoniana. Obra nunca esgotada na rica reflexão "gaddiana", tanto crítica quanto literária, I promessi sposi de Manzoni, é o livro que Gadda terá consigo in articulo mortis, uma espécie de evangelho laico que em Gadda, no entanto, é profanado e reescrito para emulação. Analisaremos especificamente como, em que forma, esta reescrita, esta imitação, esta inversão irônica da obra de Manzoni se encontra na Cognizione del dolore, mesmo à luz da complexa imbricação de vida e de trabalho, de nascimento e de escrita.

Palavras chaves: Intertextualidade. Carlo Emilio Gadda. Desleitura. Ironia. I promessi sposi. Plurilinguismo.

\section{Abstract:}

L'obiettivo di questo studio è investigare la questione delle fonti manzoniane in Carlo Emilio Gadda. Tratterò specificamente de La cogniæione del dolore (pubblicata in Brasile dall'editrice Rocco nel 1988), capolavoro di Gadda, 
pervaso da un potente pluringuismo e da un'inesauribile intertestualità. Sappiamo che Gadda era un attento lettore di Manzoni (1785-1873), al quale, già nel gennaio del 1927, sulle pagine di "Solaria" dedicava l'Apologia manzoniana. Opera inesauribile nella ricca riflessione gaddiana, I promessi sposi di Manzoni, è il libro che Gadda avrà con sé in articulo mortis, una specie di vangelo laico che Gadda, tuttavia, profana e riscrive per emulazione. Analizzeremo specificamente come, in quale forma, questa riscrittura, questa imitazione, questa inversione ironica dell'opera di Manzoni si realizza nella Cognizione del dolore, anche alla luce della complessa compenetrazione di vita e opera, di nascita e scrittura.

Parole chiave: Intertestualità. Carlo Emilio Gadda. Ironia. Dislettura. Pluringuismo. I promessi sposi.

Abstract: The objective of this study is to investigate the issue of the "manzonianas" sources in Carlo Emilio Gadda (1893-1973). I will specifically work on the Cognizione del dolore (work published by Editora Rocco in 1988: title $O$ conhecimento da dor), a masterpiece of Gadda, crossed by a powerful multilingualism, as well as an endless intertextuality. We also know that Gadda was a cultivator of Manzoni (1785-1873), to whom he dedicated the Apologia Manzoniana published in January 1927 in the pages of Solaria. Work whose wealth of literary criticism and reflection never run out according to Gada's point of view, I promessi sposi is the book that Gadda shall have with him in articulo mortis, a kind of secular gospel that in Gadda, however, is profaned and rewritten for emulation. We analyze specifically how, in what form, this rewrite, this imitation, this ironic reversal of Manzoni's work is in Cognizione del dolore, even in light of the complex intertwining of life and labor, birth and writing. 
Keywords: Intertextuality. Carlo Emilio Gadda. Reading deconstruction. Irony. I Promessi Sposi. Multilingualism.

$\mathcal{N}$ el presente studio proveremo a esplorare il tema dell'intertestualità manzoniana, segnatamente della presenza nella Cognizione del dolore di Carlo Emilio Gadda, di vari passaggi e momenti in cui si riconoscono stilemi, citazioni più o meno occulte, forme dell'elocuzione, nuclei narrativi che rimandano ai Promessi sposi. Ovviamente individuare la presenza del sottotesto manzoniano, aiuta il nostro sguardo critico a cogliere al di là delle somiglianze e dei più plausibili parallelismi, le differenze e le specificità di un testo che ha una identità forte e tratti di originalità affascinanti e unici nonché le modalità con cui questi materiali riemergono, mai puri o semplicemente citati, ma sempre "trattati" e riappropriati attraverso parodia e deformazione. L'analisi intertestuale si occuperà di riconoscere questo debito fecondo e vitalmente corrosivo per strati o livelli testuali. a) Nel sistema dei personaggi, b) Nelle forme citate e occulte che si richiamano ai manzoniani Promessi sposi, c) in alcuni espedienti stilistici utilizzati dal narratore già manzonianamente eterodiegetico, d) nella scelta dell'ambientazione latino-americana, ossia linguisticamente castigliana, in continuità letteraria con la Brianza dominata dalla Corona Spagnola di cui racconta il Manzoni. Vedremo inoltre come l'argomento scelto possa prestarsi a una lettura bloomiana che fa del Canone un terreno di conquista e di simbolica uccisione di un padre letterario: che per Gadda è appunto Manzoni. Ma diamo subito una qualche consistenza teorica a questo parallelismo biografico di cui a noi interessano unicamente le ripercussioni testuali. 
È Gianfranco Contini tra i primi critici attenti dell'opera di Carlo Emilio Gadda a parlare di una eredità manzoniana nello scrittore più giovane. Contini parla di un vero e proprio "culto" tributato da Gadda a Manzoni, che può essere spiegato da ragioni che fanno capo a una sorta di determinismo ambientale - "la comune provenienza dalla più grande città protoindustriale, poi industriale, del paese" (CONTINI, 1987) - proiettato sui caratteri affini, ovvero sulla nevrosi che accomunerebbe i due scrittori. Verrebbe da dire con Hegel, entrambi inclusi in quella categoria di "coscienza infelice borghese" che pur aderendo al capitalismo per nascita vi si oppongono per idealità. Ma certo questa lettura si presta a un anacronismo marxista che oggi suona un po' strano. Dando per superata l'intuizione marxista e appigliandoci piuttosto ai dati di fatto, di cui lo stesso Contini è prodigo, possiamo forse avanzare la convinzione che se al principio di Gadda stava Manzoni, L'apologia manzoniana scritta nel 1924 da un Gadda praticamente agli esordi, all'epilogo terreno anche, come a chiudere il cerchio. L'aneddoto continano è esemplare"il culto di Gadda per Manzoni, [che] durò fino agli ultimi giorni, quando amici si davano il cambio a leggergli il gran romanzo" (CONTINI, 1987). Vero è che questo culto, mai disonorato, può presentarsi nei termini di un'ossessione, la quale, manifestata forse un po' eccessivamente nella psicopatologia, lascerà numerose tracce nella sua scrittura, sia nelle cose realmente compiute sia in quelle incompiute, abbandonate o solo progettate. Nell'un caso come nell'altro lo spettro di Manzoni, del grande Milanese può essersi presentato a Gadda come presenza inibitrice. Procedendo su questo cammino, che non è ancora quello del testo bensì quello dell'autore, che non è ancora critico ma biografico, potremmo intersecare la teoria critica di un certo Harold Bloom. Nella Angustia da influência (BLOOM, 1991), Bloom sostiene la tesi, molto aristocratica, che i poeti forti fanno la storia della poesia (storia della poesia che è storia della letteratura tout court), "disleggendosi" (deslendo-se), in modo 
da aprire nel canone un proprio spazio di sopravvivenza. Inutile dire che c'è qualcosa di eroico e grandiosamente mitico nella concezione bloomiana, che non per niente ricorre in via allegorica al racconto miltoniano del Paradiso Perduto. Nelle intuizioni teoriche del critico americano ha un certo peso (stavo per scrivere una certa "influenza") il pensiero eterodosso di Giambattista Vico, dal quale Bloom riprende il concetto di un progressivo impoverimento del canone della letteratura mondiale. Ne consegue, inesorabilmente, che i figli saranno poeti meno grandi dei padri. L'angoscia dell'influenza è insomma il parto amaro della consapevolezza della propria inferiorità rispetto a un padre ideale. Il che comporterebbe, secondo Bloom, il tentativo frustrante e vano di riscrivere il precursore. Specificamente uno, quello più determinante, più ossessivamente necessario nella lotta per la conquista di un'identità forte. Il parricidio simbolico si compirà in termini estetici. Gadda sceglie sicuramente Manzoni. Cercheremo di analizzare come, in quali passi, in quali modi, con quali conseguenze e strategie nella Cogniz̧ione del dolore questa dislettura di Manzoni e conseguentemente questa riscrittura (o tentativo di riscrittura) del precursore ideale si dà. Tuttavia un ulteriore sforzo genericamente teorico ci risospinge verso Contini. Il principio bloomiano del post hoc ergo ante hoc, su cui Bloom ha gelosamente costruito la sua teoria dell'influenza, probabilmente rielaborando la sua formazione cabbalistica o comunque affine a certe proposte della semiosi ermetica, ha un precedente nostrano proprio in Contini. Non è forse questi a parlare in anni non sospetti (e prima di Bloom) della "Funzione Gadda" e dei "Nipotini dell'Ingegnere", ficcante formula che riassume splendidamente il concetto di influenza regrediente, con la quale $\mathrm{i}$ "poeti forti" sarebbero capaci di creare i loro precursori (in tal senso è forse impossibile al lettore moderno e postmoderno leggere Virgilio, specificamente l'Eneide, senza passare dal figlio letterario, Dante, che è diventato padre ricevendone grandiosamente l'eredità. Tantoché con Goethe riletto da Freud 
possiamo predicare "ciò che hai ereditato dai padri riconquistalo se lo vuoi possedere davvero"). Gadda insomma irradia un senso unificante su ciò che è trascorso, sulla storia letteraria italiana nei suoi esiti espressivi e mistilingui: da Cielo d'Alcamo a Folengo, dal Frugoni al Dossi e a tutta la Scapigliatura. Tuttavia mi sembra necessario premettere che la riscrittura del precursore in Gadda sceglie la strada della parodia. La parodia certamente nasce come un componimento letterario che imita in forma dissacrante un testo altrui, un'opera conosciuta (esempio celebre la parodia omerica contenuta nella Batracomiomachia), mentre tra Ottocento e Novecento la parodia come genere autonomo appare in estinzione, cedendo il posto piuttosto a un linguaggio parodico e corrosivo di cui fanno uso i grandi scrittori espressionisti/mistilingui/poligrafi da Joyce a Gadda per intenderci. Ma come esercitare la parodia su un testo bersaglio? Mi sembra che esistano due procedimenti: o la parodia si rivolge ai contenuti, che nella riscrittura vengono appunto deformati, ma preservando il linguaggio, lo stile che garantisce una riconoscibilità straniante rispetto al parodiato; oppure si esercita sul linguaggio, p.e., lo stile di Lucilio che nel II secolo a.C, imita in modo caricaturale lo stile solenne di Ennio, o ancora lo stile epico di Lucano che viene parodiato in un capitolo del Satyricon di Petronio, e ancora più recente e a noi vicino, il linguaggio petrarchesco/bembiano riprodotto da Berni in una riconoscibile caricatura dell'originale. Molto spesso la parodia stilistica si avvale della maccheronea o del pastiche. Entrambe le tecniche e i registri sono riconoscibilmente attivi nella prosa gaddiana. Per stringere il cerchio, siccome la parodia ha una parentela con umorismo e comicità, vediamo di analizzarne qui la portata e le differenze.

In termini pirandelliani, si può dire, per la modernità non è possibile arrivare al tragico o al serio se non battendo la via del faceto (PIRANDELLO, 1994). Una convinzione che ci sembra particolarmente indovinata se 
proviamo a applicarla al nostro testo di riferimento: la Cognizione del dolore. Qui, come già il titolo lascia presagire, la tragicità dell'esistenza che si sostanzia nel dolore conosciuto, esperito concretamente, appare un motivo fondante del romanzo.

Tuttavia Gadda al tragico non approda per via diretta, diciamo pure utilizzando soluzioni classiche, ma indirettamente, nelle forme mediate della comicità e dell'umorismo, della deformazione parodica, del pastiche e della nuova estetica del brutto che come Debenedetti ci insegna si collega alla crisi del personaggio nel Novecento letterario:

Appena ci si mette a leggere i romanzi moderni si è colpiti da un fatto abbastanza sconcertante. Dal ritratto dei personaggi scompare, quasi senza eccezione, ogni vestigio di bellezza fisica, specialmente nella faccia cioè nella parte più espressiva della persona. [...] In breve, col finire della narrazione naturalista $[\ldots]$ comincia nel romanzo e nel racconto l'invazione vittoriosa dei brutti, che a non lungo andare occuperanno tutto il territorio. (DEBENEDETTI, 1988).

Sotto questo aspetto la differenza tra il pienamente acclimatato al Novecento del brutto, Carlo Emilio Gadda, e quel romantico ancora ispirato, Manzoni, è stridente. Manzoni descrive i suoi personaggi semplici ma nobili, in termini di una bellezza del corpo, di un'armonia dei tratti e di una salute della persona che non possono non essere il risultato della grazia ricevuta: la bellezza interiore corrisponde a quella esteriore e per così dire la illumina. Ecco come l'autore descrive la gentile e devota Lucia mentre si abbiglia per le Nozze:

Lucia usciva in quel momento tutta attillata dalle mani della madre. Le amiche si rubavano la sposa, e le facevan forza perché si lasciasse vedere; e lei s'andava schermendo, con quella modestia un po' guerriera delle contadine, facendosi scudo alla faccia col gomito, chinandola sul busto, e aggrottando i lunghi e neri sopraccigli, mentre però la bocca s'apriva al sorriso. I neri e giovanili capelli, spartiti sopra la fronte, con una bianca e sottile dirizzatura, si ravvolgevan, dietro il capo, in cerchi moltiplici di 
trecce, trapassate da lunghi spilli d'argento, che si dividevano all'intorno, quasi a guisa de' raggi d'un'aureola, come ancora usano le contadine nel Milanese. Intorno al collo aveva un vezzo di granati alternati con bottoni d'oro a filigrana: portava un bel busto di broccato a fiori, con le maniche separate e allacciate da bei nastri: una corta gonnella di filaticcio di seta, a pieghe fitte e minute, due calze vermiglie, due pianelle, di seta anch'esse, a ricami. Oltre a questo, ch'era l'ornamento particolare del giorno delle nozze, Lucia aveva quello quotidiano d'una modesta bellezza, rilevata allora e accresciuta dalle varie affezioni che le si dipingevan sul viso: una gioia temperata da un turbamento leggiero, quel placido accoramento che si mostra di quand'in quando sul volto delle spose, e, senza scompor la bellezza, le dà un carattere particolare. (MANZONI, 1995).

Di Renzo Tramaglino, Manzoni, non ci fornisce una descrizione diretta e minuta, piuttosto ce ne appare un'immagine in movimento, dinamizzata dalle situazioni che di volta a volta si trova a vivere e superare. Sappiamo abbastanza della sua psicologia, mentre il suo aspetto sembra far tutt'uno, armonicamente, con il suo carattere: armonia e conformità tra essere e pensare, tra anima e corpo che ne rivela una certa nobiltà, nobiltà che non è più quella del sangue (che anzi può manifestarsi nelle forme degeneri di un Don Gonzalo) ma quella del giusto operare, dell'agire in conformità con il bene cristiano.

Il discorso suonerebbe opposto in Gadda. Non c’è una bellezza interiore che non sia corrotta dalla civiltà, dalle striscianti manifestazioni del conformismo. In un puro materialismo delle forme l'illuminazione interiore è stata sostituita dal cinismo e la semplicità degli umili è solo l'ennesima forma della loro ottusa ignoranza. Il catalogo della bruttezza fisica che denuncia la corruzione morale o la stolidità dei caratteri è ampissimo in Gadda e la Cogniz̧ione non fa eccezione. Iniziando dal protagonista, Gonzalo (nome già marcato dall'impronta manzoniana), che viene descritto così: “Alto, un po' curvo, di torace rotondo, maturo d'epa, colorito nel viso come un celta", ed è credo, una delle descrizioni più neutre e compiacenti che possiamo aspettarci 
da Gadda e che, non a caso, riserva al suo personaggio più amletico e dotato di tragica grandezza. Ciononostante si può osservare anche in Gonzalo l'attrazione per il brutto, e per quella corrispondenza tra interno e esterno che certo nessuna illuminazione interiore rischiara e addolcisce. La caratteristica fisica della schiena un po' curva, fa sicuramente pensare a un patimento, a un umore malinconico, come se a lui dovesse toccare la pena di sostenere il mondo sulle sue spalle. Il termine "epa" invece è già vocabolo dantesco "rispuose quel ch'avëa infiata l'epa" (DANTE, Inf. XXX, 119) e espressionisticamente carico, in una deformante. Epa, significa ventre ma anche fegato (dal greco hêpar): e a noi pare che esista la possibilità di una contaminazione tra l'organismo e il carattere, spiegata ricorrendo alla teoria medica pre-freudiana degli umori.

Ma se come dicevamo, Gonzalo spicca per la sua tragica statura che lo allontana da tutto e tutti, il catalogo delle bruttezze umane elencate con voluttà dal narratore eterodiegetico (un altro modo per chiamarsi fuori, per consentire con la distanza e la solitudine dell'individuo autocosciente) è sconfinato (qualche esempio: la Pina, il Peone, il Falegname poronga, la gottosa etc). D'altronde se individualmente si assiste a questo strazio, l'acrimonia espressionista appare anche più fanatica e esuberante nelle scene in cui la collettività è descritta come un tutto, nella bestiale stolidità del gruppo, ma forse dobbiamo dire della massa, già Novecentesca, indottrinata dalla religione degli “Odeons".

Inizierei da una dichiarazione di poetica che va letta e intesa come se dialogasse con l'altro lombardo, Manzoni. Seppur nel doppio travestimento narrativo potenziato dal transfert autobiografico che fa di Gonzalo uno scrittore mancato. Ma proprio grazie a questa invenzione il narratore, citandone il romanzo dirà: "e che il romanzo legato a dei personaggi veri e ad un ambiente vero, era stupido quanto i personaggi e l'ambiente" (GADDA, 
2011, p.731). Ecco che fine ha fatto la speranza manzoniana che il vero poetico potesse servire il vero storico, nella costruzione idealizzante di un'identità comune, di un'idea di Popolo come Nazione. Non credo sia un caso se Gadda sceglie la parola "vero" in aperta rottura con Manzoni. "Il volgo che nome non ha" non si trasformerà mai più in popolo italiano, deragliamento che sarà la conseguenza di un evento catalizzante per Gadda e per il suo immaginario: l'avvento del Fascismo. Non so quando sia stata effettivamente studiata l'infausta proiezione che Mussolini e il culto fascista della sua personalità, quintessenza dello spirito italico, abbia prodotto sulla scrittura gaddiana. Rivedere oggi i filmati dell'Istituto Luce è un po' assistere alla genesi del brutto e di una caricatura realizzata nei fatti, storicamente, a livello nazionale: il "vero" manzoniano è diventato, nella persona di Mussolini arringante le folle, caricatura ignobile e ridicola. Da questo momento in poi qualsiasi ideale di popolo unito, integro e cosciente naufraga. E la stolidità fascista, assolutamente priva di autoironia come della consapevolezza dei propri limiti diventa il marchio d'infamia di tutti i personaggi, rientranti di diritto nella categoria inglobante e praticamente ecumenica di "scemenza del mondo". Nel mondo di Gadda raramente i personaggi toccano punte di grandezza, diciamo pure di magnanimità, e perfino le cadute nell'abiezione a suo modo grandiosa quando incontrollata, sono quasi sconosciute al testo gaddiano. Vero è che tra i manzoniani Promessi Sposi e la gaddiana Cognižione c’è almeno un secolo di distanza e una rivoluzione nei gusti che non può essere sottovalutata. Con Gadda siamo in piena temperie post-verista, nel clima già tozziano e sveviano dell'anti-eroe e in generale della crisi del personaggio di debenedettiana memoria. E se per Manzoni i personaggi più grandi sono quelli in cui la nobiltà del sangue si è convertita al verbo cristiano della carità e dell'abnegazione, in una rinuncia alle ambizioni mondane per tendere agostinianamente a Dio, p.e., Fra Cristoforo, Federigo Borromeo, frate Felice 
e l'Innominato, in Gadda prevale un assoluto materialismo il quale non lascia spazio ad alcuna speranza oltramondana, dimensione anzi che condanna $i$ personaggi a una borghesissima quotidianità fatta di piccoli vizi e interessate meschinerie. Il ridicolo, lo scherno continuo, la predisposizione alla caricatura violenta, insomma, con le parole dell'autore la "sozza dipintura della mandra" (GADDA, 1931) sarebbero le armi scelte da Gadda per rappresentare i suoi mondi narrativi.

Un'altra coincidenza che rientra nel sistema dei personaggi e che non va trascurata poiché indiziale di un sottotesto possibile riferentesi ai Promessi Sposi, mi sembra sia il nome scelto per il protagonista della Cognizione: Gonzalo. Può essere che l'inferenza qui sia debole, ma non è inutile proporre anche questa suggestione di lettura. Gonzalo, è vero, è nome spagnolesco dei più comuni e tanto la Cognizione quanto i Promessi sposi si confrontano con quell'elemento culturale allotrio (ma interessante per le aperture che suscita) che è il castigliano dell'Impero per l'uno e della colonia sudamericana per l'altro, ma la rispondenza antonomastica tra i due Gonzalo, quello terribile di manzoniana memoria e il misantropo e iracondo Gonzalo della Cognizione si presta quantomeno a essere rilevata e proposta come indizio.

Varie le criptocitazioni che riguardano personaggi della Cognizione e che hanno un evidente legame con il nostro tema. Nella Cognizione a un certo punto si parla di un "agronomo che speculava il progresso" definizione che, come ha ben colto Emilio Manzotti, nasconde in realtà Alessandro Manzoni e dal quale il narratore prende evidentemente le distanze in quanto all'opportunità di usare ottimisticamente la parola "progresso": "Foi graças aos cuidados de um agrônomo que especulava o progresso e deu dele o segurro presságio, vaticinando o fim dos carvalhos, dos olmos, ou, nos fornos de cal, do antigo sonhar das faias" (GADDA, 1998, p.51). Manzoni allora, come Dio nella Bibbia, appare intradiegeticamente assieme alle sue creature, se 
è vero che un altro criptonome è quello di un tale Filarenzo Calzamaglia dietro cui non è difficile indovinare l'identità occulta nel Renzo Tramaglino, di professione filatore appunto, nonché nella circostanza in cui questo personaggio è ricordato. Mediato dal ricordo che di lui ha il governatore spagnolo Gonzalo Pirobutirro d'Eltino, discendente dell'omonimo protagonista della Cognizione, Filarenzo Calzamaglia detto Enzo, appare in una circostanza che non può non far pensare a un sostituzione voluta rispetto all'originale manzoniano:

Desonra, para ele, e pesar incurável em todo o curso sideral dos anos, foi não ter chegado em tempo para mandar enforcar em praça pública um tal de FilarenzoCalzamaglia, ou, como todos diziam, Enzo, escapulido da mão de sua justa justiça: pois tinham-lhe colocado as algemas nos pulsos durante certas desordens em San Juan, em novembro de 1688. O sujeito, entre um incêndio e outro, e depois de ficar escutando alguns cretinos tagarelando, portara-se como um idiota, além de qualquer providência imaginável [...]. (GADDA, 1998, p.48).

Gli indizi che confermano il nostro sospetto sono molti. Se infatti come dice il testo San Juan è antico nome per Pastrufazio e se Pastrufazio come ha provato la critica è Milano, la coincidenza di luogo è un primo indizio: Renzo proprio come Filarenzo viene catturato da due birri in quel di Milano, ma riesce fortunosamente a fuggire alla giustizia approfittando del clima di disordine generale conseguente ai tumulti del giorno di San Martino (la celebre rivolta per il pane), anche grazie alla presenza minacciosa di una folla di disperati ("alguns cretinos tagarelando") che impauriscono i due birri. Soltanto l'anno non coincide: 1688 per la Cognizione, 1628 per i Promessi sposi, data storicamente vera (il che potrebbe essere una svista dell'autore o un depistaggio intenzionale). Altra strizzatina d'occhio al lettore attento (o lettore-modello) è, ci pare, la parola tipicamente manzoniana "provvidenza" senza la quale risulta impossibile capire i Promessi sposi e il suo messaggio profondamente cristiano. Ma tornando a Gadda, non è priva di conseguenze 
la considerazione che il narratore voglia, attribuendo questo desiderio a un suo personaggio, impiccare sulla pubblica piazza il povero Enzo. Possiamo interpretare questa volontà come l'emersione contradditoria di amore e odio con cui Gadda sempre tratta il suo monumentale precursore.

Ciò detto, il confronto con questo patriarca della letteratura romanticorisorgimentale, nella Cogniz̧ione non si dà unicamente sul piano pur importantissimo dei personaggi, ma altresì a un livello più profondo, il quale sceglie soprattutto la parodia "citata" per mostrarsi alla superficie. Siamo insomma al punto b) della nostra ricerca sull'intertestualità delle fonti. Il momento credo più importante di questa emersione è sicuramente il celebre passaggio della Cognizione in cui, stimolato dall'immaginario doppio geografico e segnatamente orografico - il Resegone diventa il Serruchón (dallo sp. serrucho 'sega') - il narratore riscrive, ossia parodizza, "l'immortale preludio de' Promessi Sposi", operazione dichiarata e perciò stesso quasi programmatica nell'indicare il bersaglio e lo strumento:

Talché non è chi, al primo vederlo (il Serruchón) purché sia di fronte, come per esempio, di su le mura di Pastrufazio che guardano a settentrione, non lo riconosca tosto a un tal contrassegno (cioè l'andamento a sega) dalle altre Sierre di nome più oscuro e di forma più comune... (GADDA, 2011, p.721).

Lo specimen confrontato per esteso con l'originale manzoniano si rivela illuminante:

[...] talché non è chi, al primo vederlo, purché sia di fronte, come per esempio di su le mura di Milano che guardano a settentrione, non lo discerna tosto, a un tal contrassegno, in quella lunga e vasta giogaia, dagli altri monti di nome più oscuro e più comune.(MANZONI, 1995, p.16).

A un confronto più esigente la contraffazione appare evidente e ostentata. L'effetto straniante è il risultato della sostituzione dei nomi geografici e toponimici: il Serruchón appare addirittura in forma parentetica, esibizione di uno scrupolo ulteriore di cui l'autore si prende la paternità, e le 
mura ovviamente non sono più quelle di Milano bensì di Pastrufazio, città capoluogo che rappresenta il doppio in terra latino-americana di quella lombarda, come dire tanto manzoniana quanto gaddiana per nascita e memorie. Ulteriore precisazione, anche questa tra parentesi, del contrassegno del Resegone-Serruchón, che è ovviamente nel suo "andamento a sega", che completa la reticenza manzoniana, che se nel primo era un omaggio all'intelligenza dei suoi lettori, nel secondo rappresenta l'esibizione della loro patente stupidità. "La lunga e vasta giogaia" diventa nella parafrasi gaddiana "Sierre", a dare forma e espressione a uno scarto anche di natura fisica. Il Resegone per Gadda appare quasi metonimia della Brianza manzoniana, il che è certo possibile anche in virtù del suo immodificabile profilo, della sua millenaria consistenza. Tantoché lo stesso Gadda dichiarerà con la classica strafottenza del figlio per il padre: "Ogni tanto assaporo la gioia di essermi liberato dal verme solitario del Longone, con Resegone sullo sfondo e odor di Lucia Mondella nelle vicinanze" (GADDA, 2011, p.853). Manzoni per Gadda rappresenta quindi un luogo, una terra, un'origine che si vorrebbe straziare e profanare. Il luogo fisico-geografico si carica di suggestioni letterarie. In tal senso ci sembra una cautela necessaria riconoscere che ogni apertura autobiografica assorbe le plurime inferenze del letterario e che l'autore si rivela al mondo attraverso le maschere dei suoi personaggi.

Ma tornando al viluppo di temi personali, letterari e ideali, possiamo spingerci a osservare che il motivo edipico della profanazione della memoria del padre, così centrale nella Cogniżione e sul quale la critica ha giustamente insistito, nasconda un'altra profanazione, questa tutta letteraria: il figlio sul padre d'elezione, Gadda su Manzoni. Gli strumenti sono ovviamente altri: al gesto violento e iconoclasta di Gonzalo che calpesta il ritratto paterno, corrisponde quello letterario che si affida alla parodia, alla caricatura, al grottesco nonché a un'attrazione scatologica dietro cui possiamo percepire il 
segno di una rivolta contro l'autorità. Ma riprenderemo il tema più avanti, parlando del parricidio simbolico e delle implicazione testuali che ne derivano (Bloom).

Un'altro passaggio che fa esplicitamente il nome di Manzoni, seppur dall'onomastico trae un'aggettivo - che è già un caratterizzare in senso parodico il referente - è il seguente:"La lavandaia Peppa arrivò dunque a poter egutturare, con dei glu glu manzoniani da tacchino femmina [...]" (GADDA, 2011, p.719). Nel quale ci pare che l'onomatopea in questo linguaggio bestiale e inarticolato venga a contraddire con forza l'ispirazione, direi il senso etico della ricerca manzoniana, espresso in una poetica della parola che faccia della comprensibilità, della chiarezza e dell'aspirazione al "santo vero" le sue virtù proprie.

Diverso è il caso della parentela Manzoni-Gadda quando passiamo a considerare gli espedienti stilistici utilizzati dalla voce narrante eterodiegetica. Bisogna iniziare dall'uso che in entrambi gli autori è fatto delle voci in castigliano. Se infatti il ricorso a questo espediente è per certi versi analogo per soluzione, naturalmente non lo è per le ripercussioni sulle rispettive poetiche e per le implicazioni profonde sul testo, sui suoi significati, sulla posizione dell'autore rispetto al mondo dell'arte e a quello della vita. Molte delle considerazioni che verremo a trattare ci sono state offerte come spunti di lavoro dal bel saggio di Floriana di Ruzza (DI RUZZA, 2012), studiosa che si intrattiene con il problema della fonti manzoniane nella Cognizione.

Possiamo dire, preliminarmente, che se l'uso dello spagnolo in Manzoni risponde a un'esigenza di verità storica che si riflette nella sua concezione realistica di romanzo, per Gadda lo spagnolo (il maradagalese), lingua della colonia, è altresì massima espressione del barocco che abita le cose e internamente le corrompe. Insomma il castigliano è una tabe inoculata nel cuore del mondo narrativo che, ben lungi dal soddisfare criteri di verità storica 
o di un realismo di maniera, mette a soqquadro i processi di significazione e con essi la possibilitá di razionalizzazione del caotico. Ci pare anzi che tanto l'inserto di voci straniere, quanto il ricorso al dialetto, in Gadda siano sempre il sintomo di una malattia del linguaggio che si comunica alla realtà percorrendo quel nesso inscindibile che si stabilisce tra parola e cosa, tra linguaggio e mondo, tra forme di pensiero e forme di esistenza. L'onomatopea, che è ritorno alla bestialità e sottrazione assoluta della possibilità ordinatrice del mondo della vita, non si differenzia per natura sì per grado e intensità dalle altre due manifestazioni del linguaggio tipiche dei testi gaddiani, appunto il dialetto e il foresterismo. Per metonimia, se il barocco è per Gadda una categoria fondante della natura e della storia, la cui fenomenologia è percepita come "un succedersi di tentativi di ricerca, di conati, di ritrovati, d'un Arte o d'un Pensiero che trascendono le nostre attuali possibilità operative, o conoscitive, avviene fàccino a lor volta un passo falso, o più passi falsi: che nei loro conati, vale a dire nella ricerca e nell'éuresi, abbiano a incontrare la sosta o la deviazione "provvisoria" del barocco, magari del grottesco" (GADDA, 2011, p.761), allora la massima espressività di tale categoria si offre appunto nell'ipostasi dello spagnolo/castigliano in cui il barocco ha vissuto il suo massimo fulgore (il Siglo de Oro). L'ironia arguta con cui Manzoni dispone dello spagnolo nei Promessi sposi, la lingua dei dominatori, non intacca la presenza di una norma, l'italiano appunto, nella sua variante non municipalistica né dialettofona, bensì fiorentina e nazionale. I panni in Arno sono stati sciacquati per posteri, contemporanei e perfino antenati. Manzoni si diletta con l'usuale padronanza del narratore, giocando con le sottigliezze del misunderstanding. Siamo al capitolo XIII, scena in cui il governatore di Milano Antonio Ferrer, riesce a placare la folla inferocita dopo l'assalto ai forni, pronunciando un discorso che è un capolavoro d'ambiguità - 
in un politichese già maturo - e promettendo misure di giustizia, anche contro i potenti (leggi: il vicario di provvisione), che ovviamene non saranno attuate:

'Sì; si pane, pane, abbondanza; lo prometto io' e metteva la mano al petto. 'Un po' di luogo' aggiungeva subito: 'vengo per condurlo in prigione, per dargli il giusto gastigo che si merita': e soggiugeva sottovoce: 'si es culpable'. Chinandosi poi innanzi verso il cocchiere, gli diceva in fretta: 'adelante, Pedro, si puedes'.

E ancora:

'sarà gastigato... si es culpable. Sì, sì comanderò io: il pane a buon mercato. Asì es... così è, voglio dire [...] Sì, signori; pane e giustizia: in castello, in prigione, sotto la mia guardia. Grazie, grazie, grazie tante. No, no: non iscapperà! Por ablandarlos [...] Anch'io voglio bene a lor signori. Un gastigo severo. Esto lo digo por sur bien'. (MANZONI, 1995).

Diverso il discorso nel caso di Gadda. Nella Cognizione il maradagalese appare come lingua tradotta e perfino come lingua inesistente, sempre oggetto di commento metalinguistico o di verbalizzazione diretta, di riproduzione mimetica. L'intersecarsi dei linguaggi tradotti, non tradotti, dei dialetti che a loro volta appaiono e scompaiono, che sono tradotti e ritradotti in altro idioma (è il caso dello sfogo in napoletano stretto del colonnello Di Pascuale che il narratore dice avvenuto il lingua maradagalese, "salvo forse qualche battuta d'estro", confermando in pieno la sua costitutiva inattendibilità) contribuiscono all'impressione di una tale babele (il pasticcio non è solo ontologico) che sostenere che esista una lingua originaria su cui fondare un discorso comprensibile e dei valori comuni sarebbe contrario a ogni evidenza. Diciamo allora che se Manzoni ricorre all'idioma straniero per finalità mimetiche, corroborando i meccanismi di verosimiglianza storica, Gadda lo usa con tutt'altri fini. In Gadda l'emersione dell'idioma straniero ha per lo più una funzione straniante e risponde a esigenze espressionistiche, ecco perché il voluto misunderstanding italiano-spagnolo che in Manzoni permette di sfruttare il registro ironico, nella Cognizione declina indubbiamente verso il grottesco, la 
caricatura, il deforme gutturale. Insomma l'ironia ci dà il diritto di stare dalla parte del vero, mentre nella babele latino-americana della Cognizione questo privilegio si è incrinato. Ha ragione Floriana Di Ruzza quando osserva che nella Cognizione la fonetica diventa predominante rispetto alla narrazione.

Infine ci pare confermata l'esistenza di una intertestualità manzoniana nella scelta di ambientare la Cognizione in un'ipotetico stato latino-americano che in realtà riproduce le fattezze di una Brianza appunto manzoniana. Questa sovrapponibilità di luoghi tra I promessi sposi, ambientati appunto tra LeccoMonza-Milano-Brescia, agli acrocòri delle Alpi sullo sfondo e la Cogniżione, dove il riferimento travestito e straniante a una Néa Keltiké (alla lettera "Nuova Gallia" ovverosia Brianza), permette al narratore di usare forme di evidente parodia dell'originale, come nel celebre passaggio precedentemente citato nel quale sotto il travestimento toponimico si riconosceva Milano (leggi Pastrufazio), e il Resegone (leggi Serruchón). Altri ghiotti "criptonimi” nella Cognizione, tutti di pertinenza manzoniana, sono Lecco/Terepattola, Como/Novokomi, Erba/El Prado, e infine non meno importante per le ripercussioni di natura autobiografica Longone al Segrino/Lukones. A confermare la nostra tesi di una sovrapponibilità straniante tra i luoghi manzoniani e gaddiani, e per chiudere diciamo pure il cerchio, citiamo uno sfogo epistolare di Carlo Emilio a commento dell'avvenuta vendita della villa di famiglia sita appunto in quei lidi: "Ogni tanto assaporo la gioia di essermi liberato dal verme solitario Longone, con Resegone sullo sfondo e odor di Lucia Mondella nelle vicinanze".

In conclusione possiamo dire che esiste una opposizione radicale, quasi una polarità tra la concezione della lingua propria al Manzoni scrittore e intellettuale liberale, di schietti ideali Risorgimentali e quella di Gadda. Se infatti per Manzoni valse l'ideale di una lingua unitaria che doveva esprimere la nuova Nazione, lingua che, conforme all'opzione purista bembiana 
ancorché temperata verso i registri della buona favella parlata, si ispirava insomma alla lingua viva dei fiorentini, viva e contemporanea, in Gadda, sappiamo, prevalse l'assoluta negazione di quel progetto - che risultò storicamente impercorribile ( $\mathrm{e}$ in tal senso risultò molto più arguto l'Ascoli già allora avversario del Manzoni, il quale nel Proemio all'Archivio glottologico italiano proponeva la valorizzazione delle differenze naturali della lingua della nazione). Gadda porta al suo massimo splendore il filone espressionistico, appunto esaltando l'anima dialettale e la forte realtà plurilingue, centrifuga e dialettale della Penisola. Potremmo dire che di contro alle grandi idealità romantico-risorgimentali di un Manzoni, quella di Gadda è un'Italia ben rappresentata dal registro del comico-grottesco, una nazione che ha dimenticato e in parte frainteso il grande sogno politico del secolo precedente e che vive la triste passione del presente.

\section{Riferimenti Bibliografici}

BALDI, Guido. Narratologia e critica: teoria e esperimenti di lettura da Manzoni a Gadda.Napoli: Liguori, 2003.

BLOOM, Harold. A Angústia da Influência: Uma Teoria da Poesia. Rio de Janeiro: Imago Ed., 1991.

CONTINI, Gianfranco. Lo strano ingegner Gadda. In: Carlo Emilio Gadda: Romanzi e racconti. Milano: Garzanti, 2011, p. XIII.

DEBENEDETTI, Giacomo. Il personaggio nomo. Milano: Garzanti, 1988 
DI RUZZA, Floriana. L'interiorità proiettata oltreoceano: luoghi e lingue della Cognizione del dolore. Disponibile su www.ebookbrowsee.net/la/lacognizione-del-dolore. Data ultimo accesso: 02/09/2014

GADDA, Carlo Emilio. Tendo al mio fine, disponibile su: <www.gadda.ed.ac.uk/Pages/resources/essays/tendofine.php>. Data ultimo accesso: 02/09/2014.

GADDA, Carlo Emilio. 1: Romanzi e racconti. A cura di Raffaella Rodondi, Guido Lucchini, Emilio Manzotti. Milano: Garzanti, 2011.

GADDA, Carlo Emilio. O Conbecimento da Dor. Rio de Janeiro: Rocco, 1998 (tradução Mario Fondelli).

MANZONI, Alessandro. I promessi sposi. Milano: Mondadori, 1995.

PATRIZI, Giorgio. La critica e Gadda. Bologna: Cappelli Editore, 1975.

PIRANDELLO, Luigi. L'umorismo e altri saggi. Firenze: Giunti, 1994. 